\title{
Alternative Tobacco Product Use and Smoking Quit Attempts Among Teenagers in the United States: A Cross-Sectional Study
}

\author{
Colvette Brown ${ }^{1,2}$, Stanley Nkemjika ${ }^{3,4}$, Barbara Yankey ${ }^{1}$, Ike Okosun ${ }^{1}$
}

1. Population Health Sciences, School of Public Health, Georgia State University, Atlanta, USA 2. Environmental Health, Newton County Health Department, Covington, USA 3. Public Health/Epidemiology, Georgia State University, Atlanta, USA 4. Psychiatry and Behavioral Sciences, Interfaith Medical Center, Brooklyn, USA

Corresponding author: Colvette Brown, colvette.brown@gmail.com

\section{Abstract}

\section{Background}

Public health interventions have heightened awareness of risk factors and ill effects of tobacco use. Though sales of conventional tobacco products have been steadily declining, there is the advent of a new generation of alternative tobacco products marketed with claims of reduced harms and smoking cessation aids. These products are increasing in prevalence and popularity among adolescents.

\section{Aim}

The aim of this study is to assess the prevalence of tobacco quit attempts in adolescents in the United States and examine its relationship to the use and self-reported awareness of two alternative tobacco products: ecigarettes and heated tobacco products (HTPs).

\section{Methods}

This is a cross-sectional analysis of data $(2,271)$ from the 2019 National Youth Tobacco Survey (NYTS) of middle and high school students in the United States. Logistic regression analysis was employed to determine the odds of tobacco quit attempts adjusting for age, race, gender, school type, and household tobacco exposure.

\section{Results}

The overall prevalence of tobacco quit attempts among e-cigarette users and HTP users was $52.50 \%$ and $5.20 \%$, respectively. Results of multivariate regression analyses identified age ( $\mathrm{OR}=0.74,95 \% \mathrm{CI}: 0.57-0.96$ ), race $(\mathrm{OR}=1.41,95 \% \mathrm{CI}: 1.14-1.75)$, and household tobacco smoke exposure (OR=1.19, 95\% CI:1.01-1.39) as the main factors that are significantly associated with tobacco quit attempts adjusting for all other covariates.

Review began 06/15/2021 Review ended 06/30/2021 Published 07/29/2021

๑) Copyright 2021

Brown et al. This is an open access article distributed under the terms of the Creative Commons Attribution License CC-BY 4.0., which permits unrestricted use, distribution, and reproduction in any medium, provided the original author and source are credited.

\section{Conclusion}

This study did not show a statistically significant association between the awareness and use of e-cigarettes, and heated tobacco products and tobacco smoking quit attempts. Race, age, and exposure to household tobacco smoking were positively associated with quit attempts. Further studies are needed to clarify whether the use and awareness of e-cigarettes and HTPs are associated with tobacco smoking quit attempts within the US adolescent population.

Categories: Public Health, Environmental Health, Epidemiology/Public Health

Keywords: tobacco cessation, e-cigarette, alternative tobacco products, adolescents, htps

\section{Introduction}

Tobacco smoking has led to over 3 million deaths per year worldwide and 400,000 deaths per year in the US alone [1]. Today, it remains the leading cause of preventable death and disability in the US [2,3]. According to Vangeli et al., the life expectancy for smokers is reduced by three months for every year they continue to smoke over the age of 40 [4]. National anti-tobacco campaigns and other impactful public health interventions have led to increased awareness of tobacco smoking's ill effects [5]. Consequently, there has been a reduction in the sale and prevalence [6,7] of traditional combustible tobacco products (c-cigarette). However, while cigarette sales have declined, there is the emergence of a new generation of tobacco products $[8,9]$. Marketing expenditures for alternate or non-conventional tobacco products have increased in the past decade [10], which have led to products like electronic cigarettes (e-cigarette) and heated tobacco products (HTPs) increasing prevalence and popularity among smokers [11]. 
E-cigarettes are a type of electronic nicotine delivery system (ENDS) created to mimic the sensory experience of smoking traditional combustible cigarettes by vaporizing a liquid mixture that consists of propylene glycol, glycerin, flavorings, nicotine, and other chemicals [12]. It is noteworthy that e-cigarettes are commonly marketed with claims of smoking cessation [8] and have become very popular among adolescents. However, nicotine, a component of both the traditional cigarette and e-cigarette, affects adolescents' developing brains and increases the likelihood of addiction [13]. Similarly, there are additional adverse health effects of the toxic impurities in e-cigarette cartridges, for which the full extent of these health effects is still unknown [13].

The burden of disease has been increasing as 3.05 million high school students (19.3\% increase) in the United States were current e-cigarette users in 2018 [14,15]. This was an increase from $1.5 \%$ in 2011 to $20.8 \%$ in 2018. A similar trend was observed among middle school students, with an increase in e-cigarette use from $0.6 \%$ in 2011 (60,000 students) to 4.9\% (570,000 students) in 2018 [14]. The vast array of e-cigarettes has attracted young people's attention as they are available in various flavors, shapes, and colors [16]. Like ecigarettes, heated tobacco products (HTPs), also referred to as "heat-not-burn" tobacco products, have been introduced into the US market. Several concerns have been raised about the impact of these non-traditional tobacco products on youth [16]. Like e-cigarettes, HTPs utilize a mechanism whereby heat is used to volatile nicotine to a point below combustion [17], resulting in the production of an aerosol instead of burning, which produces smoke [18]. These products differ in the sense that e-cigarettes heat nicotine in a liquid solution while HTPs heat cigarette-like tobacco sticks. Tobacco companies use persuasive messages that influence the belief that HTPs are more socially acceptable compared to traditional/combustible cigarettes [9]. A study by Marynak et al. revealed that 5.2\% of the adult population in the US were aware of heated tobacco products and less than $1 \%$ reported ever use of HTPs [19].

Recently, the United States Food and Drug Authority (FDA) authorized the marketing of different HTP products in the country. Thus far, despite the approval and utilization of these products globally, only a few studies have examined the correlations between HTPs and demographic characteristics. Additionally, the impact of electronic cigarettes (e-cigarettes) and heated tobacco products (HTPs) on youth remains a controversial public health problem as it remains unclear whether alternative (non-conventional) products will lead to an uptick in the use of such products or smoking [20].

Within the public health community, there have been arguments about the viability of e-cigarettes to reduce harm or to be used by smokers to supplement or truly substitute conventional tobacco smoking and successfully attain smoking cessation $[20,21]$. The decision to use e-cigarettes as a smoking cessation intervention among youth remains conflicting; previous studies report inconsistent findings. In 2016, Kalkhoran and Glantz found that the odds of quitting tobacco smoking were $28 \%$ lower among e-cigarette users compared to non-e-cigarette users [22]. Another finding from a six-month follow-up study by Pasquereau et al. (2017) revealed that tobacco smokers who concurrently use e-cigarettes are more likely to attempt to quit at least seven times [23]. One study conducted by Kinouani et al. (2017) found that attempts to quit tobacco smoking were reported more by e-cigarette users than any other smoking group [24]. However, results from a comparative study of the differences and similarities of e-cigarette use among adolescents vs. adults showed that among young adults, e-cigarette use was not consistently associated with attempting to quit tobacco smoking [13]. Whether these factors pose a barrier to quitting among youth remains unknown. Based on the literature review, there is a dearth of studies done to examine this disparate phenomenon. Hence, our research will add to the limited body of evidence assessing the relationship between tobacco quit attempts and alternative tobacco product use among the US adolescent population.

Notably, considering that the use of non-conventional tobacco products is increasing in popularity and prevalence [21] among youth in the US [16], this has significant public health implications. It is therefore imperative that all factors that (1) present a barrier to smoking cessation within this vulnerable population are identified, and (2) examined to implement appropriate, targeted intervention(s) aimed at reducing the prevalence of use and increase the number and success of quit attempts among youth.

Hence, using a cross-sectional design, we aim to: (a) assess the prevalence of tobacco quit attempts among US adolescents in middle and high school and (b) examine the relationship between alternative tobacco product use and awareness to attempts to stop tobacco smoking. Additionally, this study sought to add to a growing body of evidence on youth tobacco-related research and bridge the literature gap.

\section{Materials And Methods Data source and study population}

The National Youth Tobacco Survey (NYTS) is an annual voluntary school-based, self-report cross-sectional survey, conducted by the CDC in collaboration with the FDA, designed to investigate tobacco-related beliefs, attitudes, behaviors, and exposure to pro-and anti-tobacco influences among public middle school (grades 6-8) and high school (grades 9-12) students. The year 2019 was the first time the survey was administered in schools using tablet computers. Participating schools determined whether parental consent would be received actively, whereby parents provided written consent allowing their child to participate in the survey, or passively, whereby parents signed and returned the consent form only if they did not want their child to 


\section{Sample selection}

A three-stage cluster sampling procedure was used to generate a nationally representative sample of U.S. students attending public and private schools in grades 6-12 from all 50 states and the District of Columbia [15]. As defined by the CDC, the primary sampling unit is a county, a group of small counties, or part of a very large county and was selected at random within each stratum. Secondary sampling units, including schools within each selected PSU, were selected randomly within each PSU [25]. At the final sampling stage, classes were selected at random within each school. Student Participation in the 2019 NYTS was voluntary and anonymous, required parental consent and student assent. From the final sample of 325 schools in 2019, 251 participated, yielding a school response rate of $77.2 \%$ (or refusal rate of $22.8 \%$ ) [25]. From a sample of 22,153 students, the total number of student questionnaires completed was 19,018, yielding a student response rate of $85.8 \%$ and an overall response rate of $66.3 \%$ [25]. Race and ethnicity were separately assessed by self-report with fixed category response options [14]. Students could select one or more of the following categories for the race: American Indian or Alaska Native, Asian, Black or African American, Native Hawaiian or other Pacific Islander, or White. Students could select whether they were Hispanic, Latino, Latina, or of Spanish origin. The National Youth Tobacco Survey (NYTS) was approved by the institutional review board of the US Centers for Disease Control and Prevention (CDC). In 2018, a pilot survey of the NYTS was conducted using two electronic versions, one programmed to align with the paperbased survey and the other to take advantage of electronic administration, including programmed skip patterns and tobacco product images [14]. The number of participants included in the 2019 dataset was 19,018 , and there were 421 variables. A detailed description of the 2019 NYTS survey design, questionnaire, and data collection can be found at https://www.cdc.gov/tobacco/data_statistics/surveys/nyts/index.htm [26].

\section{Data collection procedure}

The 2019 data collection period spanned from February 15, 2019, to May 24, 2019; during that time, a survey application was used to collect data offline that was loaded onto an electronic tablet [14]. Students absent on the day of survey administration could participate using a web-based version of the questionnaire, which was programmed to mimic the tablet-based application. In addition, skip patterns were programmed in the 2019 questionnaire to reduce respondent burden $[14,15]$. This is a secondary analysis of publicly available, de-identified data; therefore, no ethics approval was sought.

\section{Exposure assessment}

E-cigarette use was defined as using an electronic cigarette on at least one day in an entire lifetime. Selfreported awareness of e-cigarettes was assessed by the following "Do you believe that e-cigarettes are (less addictive, equally addictive, more addictive) than cigarettes? Options for answers were: Less addictive, equally addictive, more addictive, I have never heard of e-cigarettes, and I don't know enough about these products. Those who chose an option other than "I have never heard of e-cigarettes" were classified as being aware of e-cigarettes. Attempts to quit was defined as selecting (1) or more times to the question: "During the past 12 months, how many times have you stopped using all tobacco products for one day or longer because you were trying to quit all tobacco products for good?" Attempts to quit smoking tobacco products were dichotomized as yes or no based on students' responses to the previous question. Those who selected one or more times were categorized as "yes," and those who chose the answer "I did not try to quit all tobacco products during the past 12 months" were categorized as" no." Students who legitimately skipped the question were excluded.

Heated tobacco products (HTPs) self-reported awareness and use of HTPs were assessed by the questions respectively "Before today, have you ever heard of heated tobacco products? Have you ever tried a 'heated tobacco product' even just one time?"

\section{Statistical analysis}

The analyses were separately conducted for e-cigarettes and HTPs. In the descriptive statistics, the frequency distribution was reported to describe the characteristics of the population. A bivariate analysis was performed to obtain the crude odds ratio (OR) for examining the relationship between all variables in the study and tobacco quit attempts. Separate multivariate logistic regression analyses were conducted for e-cigarettes and HTPs. Factors associated with tobacco quit attempts were analyzed using logistic regression analyses. Four (4) models were estimated in the multivariate regression analyses for each study's alternative tobacco products. For e-cigarette, in the first model, tobacco quit attempt(s) (the dependent variable) was modeled with socio-demographic factors (age, race, gender, school type, age at initiation of e-cigarette). In the second model, e-cigarette use was added to the model. In the third model, e-cigarette use was substituted with self-reported awareness (of the addictiveness of ecigarette). In the final model, both e-cigarette use and self-reported awareness of the addictiveness of ecigarettes were added. The analyses were repeated for HTPs. Results were presented in the form of OR and their confidence intervals (CI). The weight, stratum, and primary sampling units (PSUs) variables provided in the public dataset were incorporated when performing analyses. All statistical data analyses were done 


\section{Cureus}

using SAS 9.4 statistical software (SAS Institute Inc.Cary, NC).

\section{Description of variables}

In this study, the dependent variable was tobacco quit attempt, dichotomized as" yes" or "no." The main independent variables of this study were assessed as binary outcomes, which include e-cigarettes use, Selfreported awareness of the addictiveness of e-cigarette, HTP use, Self-reported awareness of HTPs, and sociodemographic attributes (age, gender, race/ethnicity, school type, initiation age for e-cigarettes and household exposure status).

\section{Results}

A total of 19,018 students participated in the survey. From this sample, 2271 (11.94\%) attempted to quit tobacco smoking within the past 12 months, 18,096 (96.75\%) had heard of e-cigarettes before and were aware of their addictive nature. A total of 6,356 (33.42\%) reported the use of e-cigarettes on at least one day in their lifetime. A total of 2390 (12.57\%) and 398 (2.34\%) respondents had heard of HTP before and used HTP, respectively. Overall, $52.50 \%$ of ever-users of e-cigarettes attempted to quit tobacco smoking within the past 12 months, and the prevalence of HTP use among respondents who attempted to quit tobacco smoking within the past 12 months was $5.20 \%$.

\section{Description of the sample population}

Table 1 shows the socio-demographic characteristics of respondents in the entire study population. Of the total number of respondents (19,018), 50.74\% were non-Hispanic White, 24.38\% were Hispanic, and $12.33 \%$ were Black or African American. The majority of respondents (55.92\%) were in high school and between 13 15 years of age (44.42\%). The prevalence of household tobacco smoke exposure within the past seven days was $25.28 \%$. Approximately $97 \%$ of respondents were aware of the addictive effect of e-cigarettes, and $34.66 \%$ had used an e-cigarette on at least one day in their entire life. There was a significantly larger proportion of e-cigarette users (34.7\%) compared to HTP users (2.61\%), and only $16.52 \%$ of respondents had heard of HTPs. 


\section{Cureus}

Demographic Characteristics

Frequency

Weighted $\%(95 \% \mathrm{Cl})$

Age in years $(n=18,980)$

9-12

13-15

16-17

18 years or older

Race/Ethnicity ( $n=19,018)$

Black/African American

Hispanic

Non-Hispanic White

Other††

Gender $(n=18,902)$

Male

Female

School Type $(n=18,934)$

Middle

High

Household Tobacco smoke exposure $(n=18,613)$

Yes

No

E-cigarette use $(n=19,018)$

Yes

No

E-cigarette awareness $(n=18,704)$

Yes

No

HTP use $(n=17,031)$

Yes

No

HTP awareness $(n=14,550)$

Yes

No
3,951

8,481

5,050

1,498

2,288

5,564

8,536

2,630

9,803

9,099

8,837

10,097

4,586

14,027

6356

12662

18096

608

398

16633

2390

12160
19.200(17.131-21.269)

$44.420(42.656-46.185)$

27.883 (25.437-30.328)

8.497 (7.463-9.532)

12.334 (9.674-14.994)

24.376 (21.312-27.441)

$50.737(46.451-55.022)$

$12.553(11.055-14.051)$

52.043 (50.393-53. 693)

47.957 (46.307-49.607)

44.081 (39.779-48.383)

55.919 (51.617-60.221)

$25.280(23.336-27.224)$

74.720 (72.776-76.664

34.657 (32.536-36.779)

$65.343(63.222-67.465)$

96.993 (96.577-97.408)

$3.008(2.592-3.423)$

$2.612(1.641-3.583)$

97.388 (96.417-98.359)

$16.520(15.010-18.030)$

83.480 (81.970-84.990)

TABLE 1: Characteristics of middle and high school students in the United States, by selected demographic variables - NYTS 2019

Note: Row percent used, unweighted frequency reported. Middle school (Grades 6-8) High School (Grade 9-12).

Other $+\dagger$ (American Indian or Alaskan, Asian, Native Hawaiian or other Pacific Islander).

Bivariate logistic regression results 


\section{Cureus}

Table 2 shows the unadjusted odds of tobacco quit attempts for each study variable using bivariate logistic regression. As compared to respondents who were 13-15 years old, the odds of tobacco quit attempts were highest among adolescents between the ages of 9 and 12 ( $\mathrm{OR}=1.25,95 \% \mathrm{CI}$ : 0.897-1.754); this association was not statistically significant. Adolescents within all other age groups had lower odds of attempting to quit smoking compared to those aged 13-15 (see Table 2). Age group 16-17 (OR=0.76, CI: 0.636-0.911) or 18 years or older $(\mathrm{OR}=0.79,95 \% \mathrm{CI}: 0.635-0.974)$ was a protective factor against tobacco quit attempt. Adolescents in the age group 16-17 had 0.76 times lower odds of attempting to quit smoking compared to those in the 13-15 age group ( $\mathrm{OR}=0.76, \mathrm{CI}: 0.636-0.911)$. Individuals in the 18 years or older age category had 0.79 times (OR $=0.79$, CI: $0.635-0.974$ ) lower odds of tobacco smoking quit attempts. Notably, the bivariate analyses showed a statistically significant association between tobacco quit attempts and school type, race, age at initiation of e-cigarette, and household exposure to tobacco smoking. The odds of tobacco quit attempts were 1.36 times (OR=1.36, CI:1.13-1.63) higher among middle school students compared to high school students. The unadjusted odds of tobacco quit attempts were higher among Hispanics (OR =1.35, CI:1.10-1.66) and Black/African Americans (OR=1.15, CI:0.90-1.46) but lower among other races (OR=0.98, CI: 0.78-1.22) as compared to Non-Hispanic Whites. Adolescents exposed to tobacco smoking in the home (OR=1.25, 95\% CI:1.08-1.45), those in middle school (OR $=1.36,95 \% \mathrm{CI}: 1.13-1.63)$, those who had their first e-cigarette before age $13(\mathrm{OR}=1.39,95 \% \mathrm{CI}: 1.083-1.772)$ and females (OR=1.04, 95\% CI: 0.895-1.206) were positively linked with higher odds of attempting to quit tobacco smoking. Both e-cigarette use and HTP use were associated with greater odds of attempting to quit tobacco smoking ( $\mathrm{OR}=1.18, \mathrm{CI}: 0.90-1.54$ and $\mathrm{OR}=1.07$, 95\% CI:0.823-1.384 respectively). However, the associations were not statistically significant.

\begin{tabular}{|c|c|c|}
\hline Demographic characteristics & Odds Ratio & $95 \%$ Confidence Interval \\
\hline $9-12$ years & 1.254 & $(0.897-1.754)$ \\
\hline $13-15$ years & Reference & Reference \\
\hline 16-17 years & 0.761 & $(0.636-0.911)$ \\
\hline 18 years or older & 0.787 & $(0.635-0.974)$ \\
\hline \multicolumn{3}{|l|}{ Race/Ethnicity } \\
\hline Black/African American & Reference & Reference \\
\hline Hispanic & 1.147 & $(0.899-1.464)$ \\
\hline Non-Hispanic White & 1.353 & $(1.101-1.664)$ \\
\hline Othert† & 0.976 & $(0.780-1.221)$ \\
\hline \multicolumn{3}{|l|}{ Gender } \\
\hline Male & Reference & Reference \\
\hline Female & 1.039 & $(0.895-1.206)$ \\
\hline \multicolumn{3}{|l|}{ School Type } \\
\hline Middle & Reference & Reference \\
\hline High & 1.356 & $(1.125-1.634)$ \\
\hline \multicolumn{3}{|l|}{ Smoking characteristics } \\
\hline \multicolumn{3}{|c|}{ Household Tobacco smoke exposure } \\
\hline No & Reference & Reference \\
\hline Yes & 1.251 & $(1.076-1.454)$ \\
\hline \multicolumn{3}{|l|}{ Age at initiation of e-cigarette use } \\
\hline $9-12$ & 1.385 & $(1.083-1.772)$ \\
\hline 13-15 & 1.094 & $(0.899-1.330)$ \\
\hline $16-17$ & Reference & Reference \\
\hline 18 years and older & 0.894 & $(0.710-1.126)$ \\
\hline \multicolumn{3}{|l|}{ E-cigarette use } \\
\hline No & Reference & Reference \\
\hline
\end{tabular}




\section{Cureus}

\begin{tabular}{|c|c|c|}
\hline Yes & 1.184 & $(0.909-1.542)$ \\
\hline \multicolumn{3}{|c|}{ E-cigarette awareness } \\
\hline No & Reference & Reference \\
\hline Yes & 0.803 & $(0.512-1.259)$ \\
\hline \multicolumn{3}{|c|}{ HIP use } \\
\hline No & Reference & Reference \\
\hline Yes & 1.067 & $(0.823-1.384)$ \\
\hline \multicolumn{3}{|c|}{ HIP awareness } \\
\hline No & Reference & Reference \\
\hline Yes & 1.079 & $(0.902-1.290)$ \\
\hline
\end{tabular}

TABLE 2: Bivariate logistic regression examining the relationship between sample characteristics and tobacco quit attempts

\section{Multivariate logistic regression results for e-cigarettes}

Table 3 shows the frequency of tobacco quit attempts and the corresponding adjusted odds ratio (aOR) for all the study variables, except for HTP use and HTP awareness (assessed separately). The aORs were estimated using four different multivariate logistic regression models. Model 1 shows the aORs for the sociodemographic variables age, race, gender, school type, household exposure to tobacco smoking, and age at initiation of e-cigarette. After adjusting for all covariates, there was no longer a significant association between school type and tobacco quit attempts (aOR=1.08, 95\% CI:0.811-1.439). The adjusted estimates show that age, race, and household exposure to tobacco smoking were significantly associated with attempting to quit tobacco smoking. Adolescents within the $16-17$ age group were 0.75 times (aOR=0.75, 95\% CI: 0.578-0.972) less likely to attempt to quit tobacco smoking compared to those within the 12-15 age group. Conversely, Hispanic Whites were 1.38 times ( $\mathrm{aOR}=1.38,95 \% \mathrm{CI}: 1.110-1.711)$ as likely to attempt to quit tobacco smoking when compared with non-Hispanic Whites after adjusting for age, gender, school type, household exposure to tobacco smoking, and age at initiation of e-cigarette. The odds of tobacco quit attempt were 1.19 times (aOR=1.19, 95\% CI:1.1190-1.390) as likely among adolescents who are exposed to tobacco smoking in the home when compared to those without household tobacco smoke exposure after adjusting for age, race, gender, school type, and age at initiation of e-cigarette. All sociodemographic variables in Model 1 (age, race, gender, school type, household exposure to tobacco smoking, and age at initiation of e-cigarette) were adjusted for in subsequent models. In Model 2, e-cigarette use was added to the model to examine its relationship to a tobacco quit attempt. The adjusted estimates also showed that age, race, and household tobacco exposure were associated with a tobacco quit attempt. Hispanics were 1.39 times (aOR=1.388, 95\% CI:1.118-1.722) as likely to attempt to quit tobacco smoking compared to nonHispanic Whites, after adjusting for age, race, gender, school type, household exposure to tobacco smoking, and age at initiation of e-cigarette. Similarly, the odds of tobacco quit attempts were 1.19 (95\% CI: 1.0131.390) times as likely among adolescents exposed to tobacco smoking in their homes compared to those without household tobacco smoke exposure. See Table 3 for more results.

Participant characteristics

Participant characteristics

(\%)

Tobacco quit attempt $\mathrm{N}$

)

Age

$9-12$

13-15

16-17

18 years or older

$302(8.378)$
174 (3.890)

$977(23.939$

$816(21.158) \quad 704(18.097$
Quit attempt OR (95\% Cl)

Model $1 \quad$ Model 2

Model 3

Model 4 


\section{Cureus}

\begin{tabular}{|c|c|c|c|c|c|c|}
\hline NH-White & $\begin{array}{l}1111 \\
(31.496)\end{array}$ & $908(25.366)$ & Reference & Reference & Reference & Reference \\
\hline Black/AA & 243(6.099) & 170 (4.283) & $\begin{array}{l}1.170(0.860- \\
1.593)\end{array}$ & $\begin{array}{l}1.171(0.860- \\
1.593)\end{array}$ & $\begin{array}{l}1.156(0.849- \\
1.574)\end{array}$ & $\begin{array}{l}1.154(0.848- \\
1.572)\end{array}$ \\
\hline Hispanic & $672(14.468)$ & $431(8.610)$ & $\begin{array}{l}1.378(1.110- \\
1.711)\end{array}$ & $\begin{array}{l}1.388(1.118- \\
1.722)\end{array}$ & $\begin{array}{l}1.402(1.128- \\
1.743)\end{array}$ & $\begin{array}{l}1.411(1.136- \\
1.753)\end{array}$ \\
\hline Other & 245 (5.303) & 193 (4.374) & $\begin{array}{l}0.978(0.745- \\
1.285)\end{array}$ & $\begin{array}{l}0.992(0.755- \\
1.305)\end{array}$ & $\begin{array}{l}0.980(0.747- \\
1.287)\end{array}$ & $\begin{array}{l}0.994(0.756- \\
1.308)\end{array}$ \\
\hline \multicolumn{7}{|l|}{ Gender } \\
\hline Male & $\begin{array}{l}1239 \\
(30.093)\end{array}$ & $921(22.826)$ & Reference & Reference & Reference & Reference \\
\hline Female & $\begin{array}{l}1019 \\
(26.984)\end{array}$ & 774(19.702) & $\begin{array}{l}1.078(0.916- \\
1.268)\end{array}$ & $\begin{array}{l}1.078(0.915- \\
1.269)\end{array}$ & $\begin{array}{l}1.070(0.911- \\
1.257)\end{array}$ & $\begin{array}{l}1.070(0.910- \\
1.258)\end{array}$ \\
\hline \multicolumn{7}{|l|}{ School Type } \\
\hline High & $\begin{array}{l}1609 \\
(42.354)\end{array}$ & $\begin{array}{l}1318 \\
(33.894)\end{array}$ & Reference & Reference & Reference & Reference \\
\hline Middle & 649 (14.937) & $379(8.816)$ & $\begin{array}{l}1.081(0.811- \\
1.439)\end{array}$ & $\begin{array}{l}1.091(0.818- \\
1.455)\end{array}$ & $\begin{array}{l}1.060(0.796- \\
1.412)\end{array}$ & $\begin{array}{l}1.070(0.803- \\
1.427)\end{array}$ \\
\hline \multicolumn{7}{|c|}{$\begin{array}{l}\text { Household tobacco smoke } \\
\text { exposure }\end{array}$} \\
\hline No & $\begin{array}{l}1257 \\
(32.452)\end{array}$ & $\begin{array}{l}1049 \\
(26.662)\end{array}$ & Reference & Reference & Reference & Reference \\
\hline Yes & $953(24.676)$ & $623(16.210)$ & $\begin{array}{l}1.081(0.811- \\
1.439\end{array}$ & $\begin{array}{l}1.187(1.013- \\
1.392)\end{array}$ & $\begin{array}{l}1.187(1.015- \\
1.388)\end{array}$ & $\begin{array}{l}1.185(1.012- \\
1.387)\end{array}$ \\
\hline \multicolumn{7}{|c|}{ Age at initiation of e-cigarette } \\
\hline $9-12$ & 457 (12.376) & 269 (7.12) & $\begin{array}{l}1.038(0.757- \\
1.421)\end{array}$ & $\begin{array}{l}1.032(0.752- \\
1.415)\end{array}$ & $\begin{array}{l}1.014(0.735- \\
1.399)\end{array}$ & $\begin{array}{l}1.008(0.729- \\
1.393)\end{array}$ \\
\hline 13-15 & 725 (19.560) & 519 (14.25) & $\begin{array}{l}0.900(0.724- \\
1.119)\end{array}$ & $\begin{array}{l}0.899(0.724- \\
1.118)\end{array}$ & $\begin{array}{l}0.893(0.714- \\
1.118)\end{array}$ & $\begin{array}{l}0.893(0.714- \\
1.117)\end{array}$ \\
\hline 16-17 & 673 (19.574) & 554 (15.59) & Reference & Reference & Reference & Reference \\
\hline 18 years or older & 211 (6.096) & $189(5.431)$ & $\begin{array}{l}0.883(0.651- \\
1.197\end{array}$ & $\begin{array}{l}0.882(0.650- \\
1.197)\end{array}$ & $\begin{array}{l}0.880(0.648- \\
1.195)\end{array}$ & $\begin{array}{l}0.879(0.647- \\
1.195)\end{array}$ \\
\hline \multicolumn{7}{|l|}{ E-cigarette use } \\
\hline No & $\begin{array}{l}1528 \\
(38.419)\end{array}$ & $39(0.881)$ & ----- & Reference & ----- & Reference \\
\hline Yes & $\begin{array}{l}1529 \\
(38.419)\end{array}$ & 174 (4.215) & & $\begin{array}{l}2.609(0.918- \\
7.415)\end{array}$ & & $\begin{array}{l}2.594(0.856- \\
7.866)\end{array}$ \\
\hline \multicolumn{7}{|c|}{ E-cigarette awareness } \\
\hline No & 55 (1.4623) & $39(0.881)$ & ----- & ----- & Reference & Reference \\
\hline Yes & $\begin{array}{l}2163 \\
(55.775)\end{array}$ & $\begin{array}{l}1058 \\
(41.881)\end{array}$ & & & $\begin{array}{l}0.993(0.918- \\
7.223)\end{array}$ & $\begin{array}{l}0.189(0.468- \\
1.333)\end{array}$ \\
\hline
\end{tabular}

TABLE 3: Prevalence of tobacco quit attempt by socio-demographic characteristics, and multivariate association of the use and awareness of e-cigarette and smoking quit attempts

Note: --- Variable not included in the model, Middle school (grade 6-8), High school (Grade 9-12), NH-White (Non-Hispanic white), Black/AA (Black or African American), other (Asian, Pacific Islander, Hawaiian native, American Indian). 
Model 3 showed the adjusted odds ratios when e-cigarette use was removed from the model and replaced with e-cigarette awareness. The adjusted estimates show that age, race, and exposure to tobacco smoking in the home are significantly associated with tobacco quit attempts. After adjusting for age, race, gender, school type, household exposure to tobacco smoking, and age at initiation of e-cigarette, the likelihood of tobacco quit attempt was 1.40 times (95\% CI:1.128-1.743) as likely among adolescents who are Hispanic Whites compared to non-Hispanic Whites. The odds of tobacco quit attempts were 1.19 times (95\% CI: 1.015-1.388), as likely among adolescents living in a household with tobacco smoke exposure. There was a statistically significant association between the age group 16-17 years, and tobacco quit attempts $(\mathrm{aOR}=$ 0.742, 95\% CI:0.573-0.961). Adolescents between 16 and 17 years old were less likely to quit tobacco smoking when compared to those who are 13-15 years (See Table 2).

Model 4 showed the adjusted odds ratios for all the study variables when e-cigarette use and e-cigarette awareness were added. After adjusting for all other variables, the odds of attempting to quit tobacco smoking were 0.74 times ( $\mathrm{aOR}=0.738,95 \% \mathrm{CI}: 0.569-0.958)$ as likely among adolescents between 16 and 17 years of age compared to those aged 13-15. Similarly, race and household tobacco smoke exposure were associated with tobacco quit attempts; this was observed across all the models. Compared to non-Hispanic whites, the odds of tobacco quit attempts were 1.41 (95\% CI:1.136-1.753) as likely among Hispanics and 1.19 times (95\% CI:1.012-1.387) as likely among adolescents who are exposed to tobacco smoking in the home. Middle school students (aOR=1.07, 95\% CI: 0.80, 1.43), females ( $\mathrm{aOR}=1.07,95 \% \mathrm{CI}: 0.910-1.258)$, and those who initiated ecigarette use between the ages of 9-12 years ( $\mathrm{aOR}=1.01$, 95\% CI:0.729-1.390) had a higher odds ratio for attempting to quit tobacco smoking when compared to high school students, males and those who initiated e-cigarette use between the ages of 16-17 respectively. While the odds for attempting to quit smoking were 2.59 times (95\% CI: 0.86-7.87) as likely among e-cigarette users, it was not statistically significant; this lack of statistical significance was consistent in all the previous models.

\section{Multivariate logistic regression results for HTPs}

Table 4 shows the frequency of tobacco quit attempts and the corresponding adjusted odds ratio (aOR) for all the study variables, excluding e-cigarette use and awareness. Four different multivariate logistic regression models were created to examine the relationship between tobacco quit attempts and the use and awareness HTPs.

\begin{tabular}{|c|c|c|c|c|c|c|}
\hline \multirow[t]{2}{*}{ Participant Characteristics } & \multicolumn{2}{|c|}{ Tobacco quit attempt $\mathbf{N}(\%)$} & \multicolumn{4}{|c|}{ Quit attempt OR $(95 \% \mathrm{Cl})$} \\
\hline & Yes & No & Model 1 & Model 2 & Model 3 & Model 4 \\
\hline \multicolumn{7}{|l|}{ Age } \\
\hline $9-12$ & $174(3.890)$ & $91(2.019)$ & $\begin{array}{l}1.168(0.839- \\
1.626)\end{array}$ & $\begin{array}{l}1.510(1.010- \\
2.258)\end{array}$ & $\begin{array}{l}1.468(1.033- \\
2.087)\end{array}$ & $\begin{array}{l}1.514 \text { (1.008- } \\
2.273)\end{array}$ \\
\hline 13-15 & 977(23.939) & 645 (15.586) & Reference & Reference & Reference & Reference \\
\hline $16-17$ & $816(21.158)$ & 704 (18.097) & $\begin{array}{l}0.795(0.632- \\
1.000)\end{array}$ & $\begin{array}{l}0.766(0.591- \\
0.993)\end{array}$ & $\begin{array}{l}0.830(0.643- \\
1.072)\end{array}$ & $\begin{array}{l}0.804(0.614- \\
1.052)\end{array}$ \\
\hline 18 years or older & $302(8.378)$ & $261(6.934)$ & $\begin{array}{l}0.811 \text { (0.627- } \\
1.048)\end{array}$ & $\begin{array}{l}0.783(0.596- \\
1.028)\end{array}$ & $\begin{array}{l}0.824(0.625- \\
1.086)\end{array}$ & $\begin{array}{l}0.807(0.604- \\
1.078)\end{array}$ \\
\hline \multicolumn{7}{|l|}{ Race/Ethnicity } \\
\hline NH-White & 1111 (31.496) & $908(25.366)$ & Reference & Reference & Reference & Reference \\
\hline Black/AA & 243(6.099) & $170(4.283)$ & $\begin{array}{l}1.099(0.858- \\
1.408)\end{array}$ & $\begin{array}{l}1.190(0.854- \\
1.658)\end{array}$ & $\begin{array}{l}1.252(0.870- \\
1.802)\end{array}$ & $\begin{array}{l}1.242(0.849- \\
1.817)\end{array}$ \\
\hline Hispanic & $672(14.468)$ & $431(8.610)$ & $\begin{array}{l}1.282(1.035- \\
1.589)\end{array}$ & $\begin{array}{l}1.319(1.045- \\
1.664)\end{array}$ & $\begin{array}{l}1.249(0.966- \\
1.614)\end{array}$ & $\begin{array}{l}1.310(1.015- \\
1.690)\end{array}$ \\
\hline Other & $245(5.303)$ & 193 (4.374) & $\begin{array}{l}0.962(0.754- \\
1.227)\end{array}$ & $\begin{array}{l}0.891 \text { (0.709- } \\
1.119)\end{array}$ & $\begin{array}{l}0.952(0.747- \\
1.214)\end{array}$ & $\begin{array}{l}0.882(0.696- \\
1.117)\end{array}$ \\
\hline \multicolumn{7}{|l|}{ Gender } \\
\hline Male & $\begin{array}{l}1239(30.093) \\
921(22.826)\end{array}$ & $921(22.826)$ & Reference & Reference & Reference & Reference \\
\hline Female & $\begin{array}{l}1019 \text { (26.984) } \\
774(19.702)\end{array}$ & 774 (19.702) & $\begin{array}{l}1.078(0.916- \\
1.268)\end{array}$ & $\begin{array}{l}1.078(0.915- \\
1.269)\end{array}$ & $\begin{array}{l}1.070(0.911- \\
1.257)\end{array}$ & $\begin{array}{l}1.070(0.910- \\
1.258)\end{array}$ \\
\hline School type & & & & & & \\
\hline
\end{tabular}




\section{Cureus}

\begin{tabular}{|c|c|c|c|c|c|c|}
\hline High & 1609 ( 42.354) & $\begin{array}{l}1318 \\
(33.894)\end{array}$ & Reference & Reference & Reference & Reference \\
\hline Middle & 649 (14.937) & 379 (8.816) & $\begin{array}{l}1.068(0.823- \\
1.387)\end{array}$ & $\begin{array}{l}1.064(0.790- \\
1.437)\end{array}$ & $\begin{array}{l}1.083(0.795- \\
1.473)\end{array}$ & $\begin{array}{l}1.098(0.784- \\
1.538)\end{array}$ \\
\hline \multicolumn{7}{|c|}{$\begin{array}{l}\text { Household tobacco smoke } \\
\text { exposure }\end{array}$} \\
\hline No & 1257 (32.452) & 1049(26.662) & Reference & Reference & Reference & Reference \\
\hline Yes & $953(24.676)$ & 623(16.210) & $\begin{array}{l}1.231(1.056- \\
1.434)\end{array}$ & $\begin{array}{l}1.226(1.032- \\
1.456)\end{array}$ & $\begin{array}{l}1.200(1.003- \\
1.436)\end{array}$ & $\begin{array}{l}1.228(1.027- \\
1.468)\end{array}$ \\
\hline \multicolumn{7}{|l|}{ HTP Use } \\
\hline No & 1694 (52.122) & 128(39.033) & ----- & Reference & ----- & Reference \\
\hline Yes & 165 (5.198) & 117(3.647) & & $\begin{array}{l}0.948(0.719- \\
1.251)\end{array}$ & & $\begin{array}{l}0.908(0.599- \\
1.377)\end{array}$ \\
\hline \multicolumn{7}{|c|}{ HTP awareness } \\
\hline No & $1367(45.693)$ & $1058(34.774)$ & $\cdots$ & ---- & Reference & Reference \\
\hline Yes & $356(11.455)$ & 254 (8.078) & & & $\begin{array}{l}1.00 /(0.818- \\
1.239)\end{array}$ & $\begin{array}{l}1.001(0.11 /- \\
1.397)\end{array}$ \\
\hline
\end{tabular}

TABLE 4: Prevalence of tobacco quit attempt by socio-demographic characteristics and multivariate association of the use and awareness of HTPs and tobacco smoking quit attempts

Note: --- Variable not included in the model, Middle school (grade 6-8), High school (Grade 9-12), NH-White (Non-Hispanic white), Black/AA (Black or African American), other (Asian, Pacific Islander, Hawaiian native, American Indian).

Model 1 shows the aORs for the sociodemographic variables age, race, gender, school type, and household exposure to tobacco smoking. After adjusting for all the other variables, there was no longer a significant association between students within the 9-12 age group (aOR=1.168, 95\% CI:0.839-1.626) and tobacco quit attempts. Similarly, after adjusting for age, race, gender, and household tobacco smoke exposure, there was no longer a statistically significant association between school type (aOR $=1.068,95 \% \mathrm{CI}: 0.823-1.387)$ and tobacco quit attempts. Hispanic Whites were 1.28 times (aOR=1.282, 95\% CI: 1.035-1.589) as likely as their counterparts to make an attempt to quit tobacco smoking (non-Hispanic White) after adjusting for age, gender, school type, and household exposure to tobacco smoking. The odds of tobacco quit attempt was 1.21 times $(\mathrm{aOR}=1.231,95 \% \mathrm{CI}: 1.056-1.434)$ as likely among adolescents who are exposed to tobacco smoking in the home when compared to those without household tobacco smoke exposure, after adjusting for age, race, gender, school type, and age at initiation of e-cigarettes.

Model 2, HTP use, was added to the model to examine its relationship to tobacco quit attempts. The adjusted estimates show that adolescents aged 9-12 are 1.51 times (95\% CI:1.010-2.258) as likely to quit tobacco smoking when compared to those who are 13-15 years old. Conversely, adolescents aged 16-17 had 0.77 times (95\% CI:0.591-0.993) the odds ( $\mathrm{aOR}=0.766,95 \%$ 0.591-0.993) of tobacco quit attempt compared to those in the 13-15 age group. After adjusting for all other variables in the model, the odds of tobacco quit attempts were 1.32 times (95\% CI:1.045-1.664) as likely among Hispanic Whites compared to non-Hispanic Whites. Exposure to tobacco smoking in the home was another significant factor of tobacco quit attempts. Adolescents who live in a household where they are exposed to tobacco smoking were 1.23 times (aOR=1.226, 95\% CI:1.032-1.456) as likely to attempt to quit tobacco smoking compared to those unexposed to tobacco smoking in the home.

In Model 3, HTP use was replaced with HTP awareness. After adjusting for all covariates, the odds of tobacco quit attempt were 1.47 times ( $\mathrm{aOR}=1.468,95 \% \mathrm{CI}: 1.033-2.087$ ) as likely among those between the ages of 912 compared to those who are 13-15 years old. Notably, the odds of tobacco quit attempts decreased with increasing age when HTP awareness was added to the model. The aOR for adolescents between 16-17 years old was 0.830 , 95\% CI:0.643-1.072 and for those 18 years or older, aOR $=0.824$ (95\% CI:0.625-1.086). There was a statistically significant association between household tobacco smoke exposure and tobacco quit attempts. The odds of tobacco quit attempts were 1.20 times (95\% CI: 1.003-1.436) as likely among adolescents living in a household with tobacco smoke exposure. There was no statistically significant association between HTP awareness ( $\mathrm{aOR}=1.007,95 \% \mathrm{CI}: 0.818-1.239$ ) and tobacco quit attempts. 
quit attempt decreased with increasing age after adjusting for age, race, gender, school type, and household tobacco exposure. After adjusting for other variables, the odds of tobacco quit attempt were 1.51 times $(\mathrm{aOR}=1.514,95 \% \mathrm{CI}: 1.008-2.273)$ as likely among adolescents aged 9-12 compared to those aged 13-15. Hispanic adolescents were more likely to have made a quit attempt compared to non-Hispanic White adolescents (aOR=1.310, 95\% CI:1.015-1.690) after adjusting for all other variables. Similarly, adolescents exposed to tobacco smoke in the home were 1.23 times (95\% CI:1.027-1.468) as likely to attempt to quit tobacco smoking compared to their peers who are unexposed to tobacco smoking in the home. There was no statistically significant association between HTP use or awareness, and tobacco quit attempt (aOR= 0.91 ; 95\% CI:0.599-1.377) and (aOR=1.00; 95\% CI: 0:717-1.397) respectively.

Table 5 shows the unadjusted and adjusted odds of tobacco quit attempts among users of both HTPs and ecigarettes. As compared to non-HTP users, the odds of smoking quit attempts were 0.88 times (95\% CI: 0.572-1.351) as likely among HTP users after adjusting for age, race, gender, school type, e-cigarette awareness (addictive property), and household exposure to tobacco smoking which was not statistically significant. Conversely, the odds of tobacco quit attempts were 1.17 times (95\% CI: 0.869-1.581) as likely among e-cigarette users compared to non-e-cigarette users after adjusting for all other covariates, which were also not statistically significant.

\begin{tabular}{|c|c|c|c|}
\hline Characteristics & Unadjusted OR (95\% Cl) & Adjusted OR (95\% Cl) & P-value \\
\hline \multicolumn{4}{|l|}{ HTP use †† } \\
\hline Yes & $1.067(0.727-1.351)$ & $0.879(0.572-1.351)$ & 0.55 \\
\hline No & Reference & Reference & \\
\hline \multicolumn{4}{|l|}{ E-cigarette use†† } \\
\hline Yes & $1.184(0.909-1.542)$ & $1.172(0.869-1.581)$ & 0.29 \\
\hline No & Reference & Reference & \\
\hline
\end{tabular}

\section{TABLE 5: Unadjusted and adjusted OR for the relationship between the combined use of e-} cigarettes and HTPs and tobacco quit attempts

††Adjusted for age, race, gender, school type, e-cigarette awareness, and household exposure to tobacco smoking.

OR: Odds ratio

\section{Discussion}

Based on the results of our study, there is an overall higher prevalence of tobacco quit attempts among adolescents between 13-15 years old (23.9\%), non-Hispanic Whites (31.5\%), males (30.1\%), high school students (42.4\%), and among e-cigarette users (52.5\%). Notably, a higher proportion of e-cigarette users attempted to quit smoking than their non-e-cigarette-using counterparts ( $52.5 \%$ vs. $38.4 \%)$. This finding is consistent with results from a study conducted in Korea by Kang and Cho (2019), which investigated the prevalence and association of HTP use [27].

We hypothesized that the use and awareness of e-cigarettes and HTPs were strongly associated with a tobacco quit attempt. However, despite numerous advertising and media messages claiming e-cigarettes to be a safer alternative to c-cigarettes and as a smoking cessation aid, findings from our study showed no statistically significant association between e-cigarette use and tobacco quit attempts among adolescents in the US, after adjusting for all covariates. This finding was consistent with studies done in Finland [8], Canada [11], and the US [13,28,29], which found no association between e-cigarette use and tobacco smoking cessation among adolescents and adults. Similarly, our analyses based on gender stratification (result not shown) showed no association between e-cigarette use and tobacco quit attempts ( $\mathrm{aOR}=1.01,95 \% \mathrm{CI}$ : 0.701.463, $\mathrm{P}=0.9518$ ). Additionally, our study showed no statistical significance between the self-reported awareness of the addictiveness of e-cigarettes and tobacco quit attempts within the past year. This finding was also consistent with a 2014 study conducted in the US, which reported no significant association between e-cigarette awareness and past-year quit attempts [20]. However, our study's findings conflicted with the results of a longitudinal study that revealed that e-cigarette use for cessation was associated with increased odds of smoking cessation at six and 12 months follow-up compared to non-users of e-cigarettes [23].

A possible explanation for that finding may stem from the influence of the social and physical environment on one's health decisions and behaviors. The majority of participants in this study noted that the two main 
reasons for their use of e-cigarettes were: (a) they were being used by a friend or family member, (b) they were curious about them. It is, therefore, possible that adolescents may have begun using these products because they are trendy, popular, publicly available [11], or as a consequence of the influence of peers, family members, and advertisements (a risk factor for use) and not to aid in tobacco quit attempts [15]. Also, the ability to experiment with the nicotine content in electronic cigarettes may increase the likelihood of addiction, leading to increased use but no benefit of smoking cessation. In 2013, Sutfin et al. found that $12 \%$ of e-cigarette users had never smoked a conventional cigarette or any other tobacco product. Sutfin et al.'s study may indicate that adolescents use e-cigarettes to substitute for traditional tobacco (combustible cigarette) rather than as a smoking cessation aid [28]. Another explanation for the lack of association may be a consequence of the cross-sectional study design. There may not have been an adequate amount of time in between adolescents becoming aware of the addictive properties of e-cigarettes and their decision to quit tobacco smoking [13]. Although this study did not examine poly-tobacco use, research shows that it is more difficult for tobacco smokers to quit smoking when using alternative tobacco products and multiple conventional tobacco products concurrently [30]. Therefore, the possibility exists that e-cigarette users in this study also used various tobacco products, making it more difficult for them to quit. Also, how e-cigarette use (one of the independent variables) was defined differs between studies. In this study, e-cigarette use was defined as using an e-cigarette on at least one day in the entire lifetime. In contrast, e-cigarette use was defined by Pasquereau et al. (2017) as regular use of e-cigarettes within the past 30 days [23].

Our study also showed no significant association between tobacco quit attempts with the use and awareness of HTPs, respectively. This finding was consistent with the literature on studies done in both US and abroad, demonstrating no correlation between HTP use and awareness with tobacco quit attempts (CHOICESTRATA) [23]. Our study suggests that age, race, and household exposure to tobacco smoking were significantly associated with conventional tobacco smoking quit attempts with regards to sociodemographic characteristics. Additionally, the burden of evidence shows that there are disparities among racial/ethnic groups in relation to smoking behaviors, such as quit attempts [6,31]. Our study supports this evidence as there were notable differences in tobacco quit attempts among different racial/ethnic groups. Hispanic Whites were more likely to attempt quitting tobacco smoking when both e-cigarette use and awareness and HTP use and awareness were examined in our analysis (OR=1.41, 95\% CI:1.14-1.75 and OR=1.31, 95\% CI:1.02-1.36 respectively). This finding was consistent with a 2011 study that reported significant quit attempts correlates with race/ethnic group, which found that Hispanics were more likely to have made tobacco quit attempts than Whites $[6,30,31,32]$.

Our study also showed a positive association between tobacco quit attempts and household exposure to tobacco smoking. This finding supports a similar result from several studies conducted in 14 countries [33], as exposure to smoking in the home was positively associated with attempting smoking cessation. The ecological model of health behavior could explain this result in social and environmental interactions, which can influence smoking behaviors among adolescents [22]. Abrantes et al. (2009) reported that a generalized belief of tobacco's adverse health-related sequelae is related to attempting to quit. Adolescents in this study exposed to tobacco smoking at home may have observed firsthand the negative implications of smoking on one's health [5]. They may have had to become caregivers to family members who have become disabled due to tobacco smoking, or they may have lost a family member from illnesses associated with tobacco use.

Another finding from our study showed an overall lower odds ratio for tobacco quit attempts for older adolescents compared to younger adolescents; this is consistent with the results of a study by Abrantes et al. 2009 [5]. Biological nicotine naivety among younger smokers has been attributed to this finding [34]. We also demonstrated that e-cigarettes (34.7\%) are more prevalent among middle and high school students than HTPs (2.6\%). This result was similar to findings from studies conducted in Japan [34], Korea [27], and the US [32]. Notably, HTP use in Japan had increased tenfold within two years of introduction to the Japanese market [34]. Evidence in the literature suggests that HTP was projected to surpass e-cigarettes, which had increased 20-fold within three years of introduction in the Korean market [27]. Therefore, it is imperative that though HTP prevalence among adolescents is presently lower than e-cigarettes in the US, there needs to be monitoring and surveillance of this product, particularly within the adolescent population, to identify use patterns and to implement fast and proactive control measures to prevent epidemic rates. While there was no statistical significance between the use and awareness of HTP or the use and awareness of e-cigarettes and smoking, quit attempt, a biological/clinically significant association may exist.

The marketing claims of tobacco companies regarding the benefit of alternative tobacco products such as smoking cessation aid remain controversial [35]. A literature review shows a lack of consistency about the positive association between tobacco smoking quit attempts and the use of alternative tobacco products. A previous study by Kalkhoran and Glantz from 2016 found that the odds of discontinuing tobacco smoking were $28 \%$ lower among e-cigarette users compared to non-e-cigarette users [22]. Similarly, the results of a comparative study by Carroll Chapman and $\mathrm{Wu}$ (2014) showed that e-cigarette use was not consistently associated with a tobacco quit attempt [13]. Studies in support of the lack of association between e-cigarette use and tobacco quit attempts include studies done in Finland [8], Canada [17], and the US [36]. However, Kinouani et al. (2017) reported that smoking quit attempts were informed more by e-cigarette users than any other smoking group [24]. Also, results from a six-month follow-up study revealed that e-cigarette users had a higher likelihood of attempting to quit smoking at least seven times than non-e-cigarette users [28]. 


\section{Strengths and limitations}

There are some strengths and weaknesses of this study that may have implications in interpreting the results. The strengths of this study are (1) the data is from the National Youth Tobacco Survey, a nationally representative sample of middle and high school students in the US, hence representative in scope; (2) the questionnaires and measurements in the National Youth Tobacco Survey were done using excellent techniques. The training and quality control measures of the National Youth Tobacco Survey give added reliability to the data and the results of this study; (3) it provides an understanding of the prevalence of tobacco quit attempts by sociodemographic characteristics among middle and high school students in the US.

There are some limitations of this study that need to be noted. The data was cross-sectional, allowing no causal conclusions since it is not known whether the factors that were examined preceded the outcome. In this study, respondents provided information that might be subject to reporting bias; thus, the true prevalence of tobacco quit attempts may be under-reported. This study also did not consider the possibility of concurrent use of other cessation aids such as nicotine patches, sprays, etc. Future studies to compare the prevalence of quit attempts between individuals who are solely using alternative tobacco products as cessation aids and individuals using a combination therapy should be conducted to provide further insight into the factors associated with a quit attempt.

\section{Public health practice and policy implications}

The results of this study highlight the importance of comprehensive, efficacious public health interventions. A strong focus should be placed on tailoring interventions to the adolescent population, including health education and promotion geared towards increasing awareness of the deleterious effects of alternative tobacco product use. Tobacco health information must be communicated in plain language and should consider the health literacy level of the target population; this allows the message to be easily understood and interpreted. School-based interventions may play a critical role in reducing the appeal and acceptability of alternative tobacco products. One recommendation for intervention is to incorporate tobacco and substance use education into the school curriculum. This will provide an avenue through which adolescents will become more knowledgeable about the implications of tobacco use, thereby allowing them to make more informed decisions about the use of tobacco products.

Additionally, providing free tobacco cessation counseling at school and incentivizing students to participate in interscholastic competitions to deter tobacco product use and promote tobacco quit attempts may prove effective at mitigating the risks associated with tobacco use. At the national level, implementing strategies to reduce the availability and marketing of alternative tobacco products should be considered. One approach that can be taken to reduce the prevalence and use among adolescents is to increase the taxes and sales price of these products, making them less affordable for adolescents. In addition, healthcare providers may implement tobacco screening for adolescents in the clinical setting to assess tobacco use behaviors and provide necessary resources to successfully achieve smoking cessation [35]. Given that the result of this study revealed that race/ethnicity was associated with tobacco quit attempts, it is imperative to implement racial-ethnic specific culturally tailored interventions to increase self-efficacy and belief about the healthrelated consequences of tobacco use. Such an intervention may lead to higher rates of quitting.

\section{Conclusions}

This study showed no association between conventional tobacco quit attempts with the awareness and use of e-cigarettes and HTP. However, race, age, and exposure to household tobacco smoking were positively associated with a quit attempt. The lack of association between the use and awareness of e-cigarette and HTPs warrants the need for a more robust prospective study to determine the true nature of the relationship between use and awareness of alternative tobacco products and quit attempts. Future studies using a prospective study design may provide more useful information about quit attempts and explore the differences in successful quit attempts among different sociodemographic groups within the U.S. adolescent population. Finally, future interventional approaches may consider parental or guardians' knowledge of ecigarettes and HTPs, which may serve as a foundation to advocate for changes to national e-cigarette and HTP regulation policies.

\section{Additional Information \\ Disclosures}

Human subjects: Consent was obtained or waived by all participants in this study. Animal subjects: All authors have confirmed that this study did not involve animal subjects or tissue. Conflicts of interest: In compliance with the ICMJE uniform disclosure form, all authors declare the following: Payment/services info: All authors have declared that no financial support was received from any organization for the submitted work. Financial relationships: All authors have declared that they have no financial relationships at present or within the previous three years with any organizations that might have an interest in the submitted work. Other relationships: All authors have declared that there are no other relationships or activities that could appear to have influenced the submitted work. 


\section{References}

1. McGoldrick DE, Boonn AV: Public policy to maximize tobacco cessation . Am J Prev Med. 2010, 38:S327-32. 10.1016/j.amepre.2009.11.017

2. Ahluwalia IB, Smith T, Arrazola RA, et al.: Current tobacco smoking, quit attempts, and knowledge about smoking risks among persons aged $\geqslant 15$ years-Global Adult Tobacco Survey, 28 countries, 2008-2016. MMWR Morb Mortal Wkly Rep. 2018, 67:1072-6. 10.15585/mmwr.mm6738a7

3. Shete SS, Wilkinson AV: Identifying demographic and psychosocial factors related to the escalation of smoking behavior among Mexican American adolescents. Prev Med. 2017, 99:146-51. 10.1016/j.ypmed.2017.02.018

4. Vangeli E, Stapleton J, Smit ES, Borland R, West R: Predictors of attempts to stop smoking and their success in adult general population samples: a systematic review. Addiction. 2011, 106:2110-21. 10.1111/j.13600443.2011.03565.x

5. Abrantes AM, Lee CS, MacPherson L, Strong DR, Borrelli B, Brown RA: Health risk behaviors in relation to making a smoking quit attempt among adolescents. J Behav Med. 2009, 32:142-9. 10.1007/s10865-008-91841

6. Kahende JW, Malarcher AM, Teplinskaya A, Asman KJ: Quit attempt correlates among smokers by race/ethnicity. Int J Environ Res Public Health. 2011, 8:3871-88. 10.3390/ijerph8103871

7. Dai H: Heated tobacco product use and associated factors among U.S. youth, 2019 . Drug Alcohol Depend. 2020, 214:108150. 10.1016/j.drugalcdep.2020.108150

8. Kinnunen JM, Ollila H, El-Amin Sel-T, Pere LA, Lindfors PL, Rimpelä AH: Awareness and determinants of electronic cigarette use among Finnish adolescents in 2013: a population-based study. Tob Control. 2015, 24:e264-70. 10.1136/tobaccocontrol-2013-051512

9. Lempert LK, Glantz SA: Heated tobacco product regulation under US law and the FCTC . Tob Control. 2018, 27:s118-25. 10.1136/tobaccocontrol-2018-054560

10. Messer K, Vijayaraghavan M, White MM, et al.: Cigarette smoking cessation attempts among current US smokers who also use smokeless tobacco. Addict Behav. 2015, 51:113-9. 10.1016/j.addbeh.2015.06.045

11. Malas M, van der Tempel J, Schwartz R, et al.: Electronic cigarettes for smoking cessation: a systematic review. Nicotine Tob Res. 2016, 18:1926-36. 10.1093/ntr/ntw119

12. Zhong J, Cao S, Gong W, Fei F, Wang M: Electronic cigarettes use and intention to cigarette smoking among never-smoking adolescents and young adults: a meta-analysis. Int J Environ Res Public Health. 2016, 13:465. 10.3390/ijerph13050465

13. Carroll Chapman SL, Wu LT: E-cigarette prevalence and correlates of use among adolescents versus adults: a review and comparison. J Psychiatr Res. 2014, 54:43-54. 10.1016/j.jpsychires.2014.03.005

14. Cullen KA, Gentzke AS, Sawdey MD, et al.: e-cigarette use among youth in the United States, 2019. JAMA. 2019, 322:2095-103. 10.1001/jama.2019.18387

15. Gentzke AS, Wang TW, Marynak KL, Trivers KF, King BA: Exposure to secondhand smoke and secondhand ecigarette aerosol among middle and high school students. Prev Chronic Dis. 2019, 16:E42. 10.5888/pcd16.180531

16. Wang B, King BA, Corey CG, Arrazola RA, Johnson SE: Awareness and use of non-conventional tobacco products among U.S. students, 2012. Am J Prev Med. 2014, 47:S36-52. 10.1016/j.amepre.2014.05.003

17. Czoli CD, White CM, Reid JL, OConnor RJ, Hammond D: Awareness and interest in IQOS heated tobacco products among youth in Canada, England and the USA. Tob Control. 2020, 29:89-95. 10.1136/tobaccocontrol-2018-054654

18. Dunbar MS, Seelam R, Tucker JS, Rodriguez A, Shih RA, D'Amico EJ: Correlates of awareness and use of heated tobacco products in a sample of US young adults in 2018-2019. Nicotine Tob Res. 2020, 22:2178-87. 10.1093/ntr/ntaa007

19. Marynak KL, Wang TW, King BA, Agaku IT, Reimels EA, Graffunder CM: Awareness and ever use of "heatnot-burn" tobacco products among U.S. Adults, 2017. Am J Prev Med. 2018, 55:551-4. 10.1016/j.amepre.2018.04.031

20. Tan AS, Bigman CA: E-cigarette awareness and perceived harmfulness: prevalence and associations with smoking-cessation outcomes. Am J Prev Med. 2014, 47:141-9. 10.1016/j.amepre.2014.02.011

21. CDC. Youth and tobacco use. (2020). Accessed: July 30, 2021: https://www.cdc.gov/tobacco/data_statistics/fact_sheets/youth_data/tobacco_use/index.htm.

22. Kalkhoran S, Glantz SA: E-cigarettes and smoking cessation in real-world and clinical settings: a systematic review and meta-analysis. Lancet Respir Med. 2016, 4:116-28. 10.1016/S2213-2600(15)00521-4

23. Pasquereau A, Guignard R, Andler R, Nguyen-Thanh V: Electronic cigarettes, quit attempts and smoking cessation: a 6-month follow-up. Addiction. 2017, 112:1620-8. 10.1111/add.13869

24. Kinouani S, Pereira E, Tzourio C: Electronic cigarette use in students and its relation with tobacco-smoking: a cross-sectional analysis of the i-Share study. Int J Environ Res Public Health. 2017, 14:1345. 10.3390/ijerph14111345

25. Wang TW, Gentzke AS, Creamer MR, et al.: Tobacco product use and associated factors among middle and high school students - United States, 2019. MMWR Surveill Summ. 2019, 68:1-22. 10.15585/mmwr.ss6812a1

26. CDC. National Youth Tobacco Survey (NYTS). (2020). Accessed: July 29, 2021: https://www.cdc.gov/tobacco/data_statistics/surveys/nyts/index.htm.

27. Kang H, Cho SI: Heated tobacco product use among Korean adolescents. Tob Control. 2020, 29:466-8. 10.1136/tobaccocontrol-2019-054949

28. Sutfin EL, McCoy TP, Morrell HE, Hoeppner BB, Wolfson M: Electronic cigarette use by college students . Drug Alcohol Depend. 2013, 131:214-21. 10.1016/j.drugalcdep.2013.05.001

29. Mantey DS, Cooper MR, Loukas A, Perry CL: E-cigarette use and cigarette smoking cessation among Texas college students. Am J Health Behav. 2017, 41:750-9. 10.5993/AJHB.41.6.9

30. Popova L, Ling PM: Alternative tobacco product use and smoking cessation: a national study . Am J Public Health. 2013, 103:923-30. 10.2105/AJPH.2012.301070

31. Li H, Hansen AR, McGalliard Z, Gover L, Yan F, Zhang J: Trends in smoking and smoking cessation during 


\section{Cureus}

pregnancy from 1985 to 2014, racial and ethnic disparity observed from multiple national surveys. Matern Child Health J. 2018, $22: 685-93.10 .1007 /$ s10995-018-2437-x

32. Chithambo TP, Huey SJ: Black/white differences in perceived weight and attractiveness among overweight women. J Obes. 2013, 2013:320326. 10.1155/2013/320326

33. Okoli CT, Kodet J: A systematic review of secondhand tobacco smoke exposure and smoking behaviors: Smoking status, susceptibility, initiation, dependence, and cessation. Addict Behav. 2015, 47:22-32. 10.1016/j.addbeh.2015.03.018

34. Tabuchi T, Kiyohara K, Hoshino T, Bekki K, Inaba Y, Kunugita N: Awareness and use of electronic cigarettes and heat-not-burn tobacco products in Japan. Addiction. 2016, 111:706-13. 10.1111/add.13231

35. Marshall JR, Lotfipour S, Chakravarthy B: Growing trend of alternative tobacco use among the nation's youth: a new generation of addicts. West J Emerg Med. 2016, 17:139-42. 10.5811/westjem.2016.1.29383

36. Nyman AL, Weaver SR, Popova L, Pechacek TF, Huang J, Ashley DL, Eriksen MP: Awareness and use of heated tobacco products among US adults, 2016-2017. Tob Control. 2018, 27:s55-61.

10.1136/tobaccocontrol-2018-054323 